\title{
Visible Light Communication Based Traffic Information Broadcasting Systems
}

\author{
Navin Kumar
}

\begin{abstract}
Traffic safety information broadcast from traffic lights using Visible Light Communication (VLC) is a new cost effective technology which can draw attention to drivers to take necessary safety measures. This paper presents a VLC broadcast system considering LED-based traffic lights. It discusses the conceptual methodology for integrating traffic light road side unit (RSUs) with impending intelligent transportation systems (ITS) architecture. Results from a case study of VLC system for information broadcast are also presented.
\end{abstract}

Index Terms-Visible light communication, intelligent transportation systems, LEDs.

\section{INTRODUCTION}

Road accidents which cause loss of material and most importantly human lives are becoming severe even with the deployment of many intelligent communication devices on board vehicle and alongside the road. According to world health organization report [1] road crashes are the second leading cause of death globally among young people aged five to 29 and the third leading cause of death among people aged 30 to 44 years. Over 1.2 million people are killed annually because of road accidents. The study predicted that road accidents would become the sixth largest cause of death in the world in 2020 whereas it was the ninth largest cause of death in 1990.

Various modes of vehicular communications, such as vehicle-to-infrastructure (V2I), vehicle-to-vehicle (V2V) and infrastructure-to-vehicle (I2V), are being investigated to reduce road accidents and fatalities. The emergence of the IEEE $802.11 \mathrm{p}$ standard [2] for short to medium range inter-vehicle communication and the allocation of a dedicated frequency band for ITS communication in Europe have paved the way for future implementations of communication-based ITS safety applications [3]. ITS, which interrelates humans, roads, and vehicles through state-of-art Information Technology (IT) are new transport systems for the purpose of the solution of the road transportation problems, aiming for efficient traffic flow and reduction of the environmental load. ITS is directly related to human and material safety. Recently, ITS have drawn a lot of attention to solve various traffic problems.

Projects such as PREVent [4], and CALM [5] are being investigated and realized worldwide to reduce road fatalities. Visible light communication based project VIDAS (VIsible light communication for advanced Driver Assistant Systems)

Manuscript received December 29, 2012; revised April 12, 2013. This work was supported in part by FCT Grant under project VIDAS.

Navin Kumar is with CMRIT, Bangalore, India, 560078 (e-mail: navin_kum3@yahoo.com). is another challenging project being realized to minimize such losses. This is particularly interesting as it uses existing infrastructures, resulting in low cost communication systems by exploring traffic lights as road side units based on VLC. VLC is normally based on light emitting diode (LED) which has many advantages such as highly energy efficient, long life, harmless to human and friendly to environment (leads green technology).

LED-based VLC [6] systems can be deployed in vehicular environment on existing infrastructure such as LEDs traffic signal lights. There are many high priority road safety applications and services of VLC systems in vehicular environment. All of them assist drivers making safe decisions in traffic and complying with traffic regulations. The VLC systems can broadcast road traffic safety information in real time or pre-recorded, minimizing the possible accidents and increasing smooth flow of traffic on road. Furthermore, LED-based road lights can offer ubiquitous road to vehicle communication (URVC) throughout travel.

This paper presents an information broadcasting system (in I2V mode) based on LED visible light in which LED-based traffic lights used as RSU. A VLC system's architecture suitable for above application is discussed in brief. The concept of VLC system integration with RSUs and ITS architecture is briefly introduced. A prototype demonstration of VLC and some of the experimental results from a case study are presented to highlight the suitability of the system in intended applications.

Rest of the contents in the paper is given as follows. Section II describes VLC system intended for ITS applications. Overall VLC broadcast system architecture is presented in section III, while experimental scenario and relevant results are outlined in section IV. Section V concludes the paper.

\section{VLC IN ITS}

VLC systems have many important and novel applications. They can play a key role in ITS from broadcasting important traffic information to $\mathrm{V} 2 \mathrm{~V}$ and car2infrastructure (V2I) communication facilities. Basic study on traffic information system using LED traffic lights was carried out and analyzed in paper [6]. Authors in [7] analyzed VLC for ITS using 2-dimensional image sensor.

LED-based traffic lights are well suited for broadcast communication in $\mathrm{I} 2 \mathrm{~V}$ mode of vehicular communication systems. The light emitted from a traffic light (consisting of an array of LEDs) is modulated at a frequency undetectable to human eyes. The modulated light is then detected by a photo diode (PD) based receiver on the vehicle, providing useful safety information to the driver in advance. 


\section{A. VLC into ITS Architecture}

LED-based traffic lights offer very suitable option to be included as RSU. This system can be integrated with ongoing development of ITS architecture. One of the suitable scenarios is presented in Fig. 1. Traffic information picked up in real time or prerecorded is processed through traffic controller. Based upon the signal, the information passes through VLC transmitter system to traffic light which finally emits the data along with signaling. There are many access technologies proposed in ITS architecture. LED-based VLC can also be used. Additional monitor unit can be included to support information in conjunction with other RSUs. In this systems therefore, integrating LED-based traffic light unit for broadcasting safety related information offer a cost effective method of implementation. For this case, the system offers dual function of LED; signaling and traffic broadcast unit. VLC therefore can be considered as supplementary communication systems.

ITS in fact, use a number of technologies and many more are likely to be used. In this situation, it becomes very important for an ITS architecture to be flexible enough to accommodate integration of new systems. Following ITS architectural framework [8], VLC reference protocol architecture is developed.

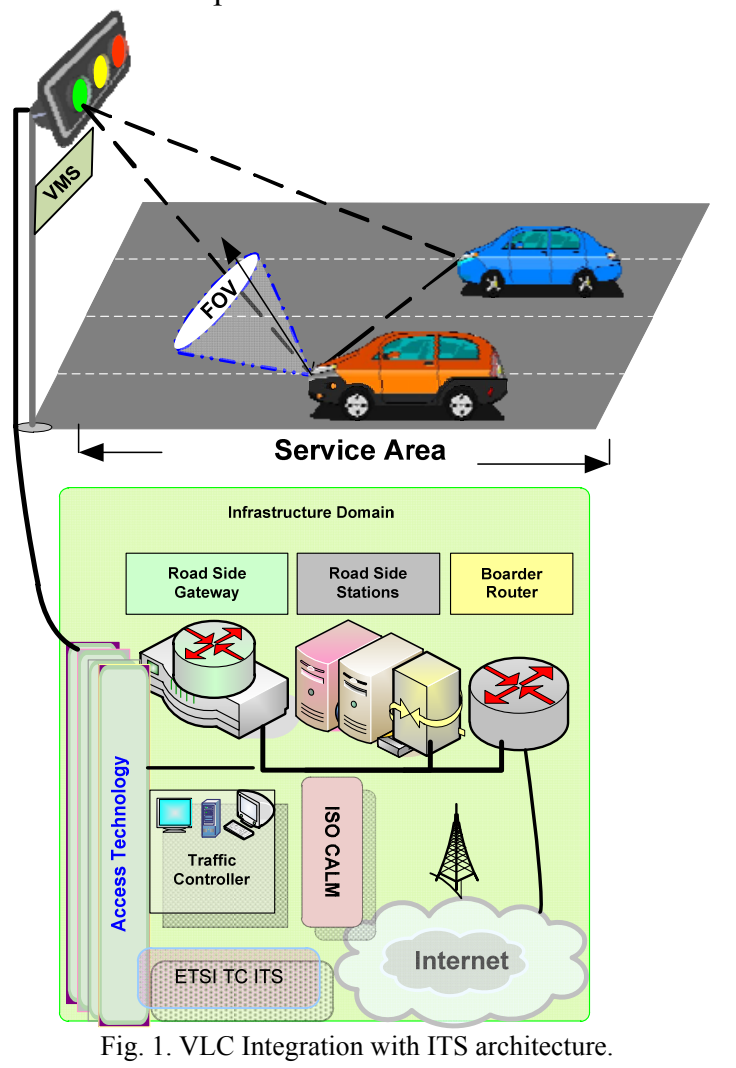

\section{B. VLC Reference Model}

A layered architecture for VLC communication reference model is shown in Fig. 2. The reference protocol architecture basically obeys the ISO/OSI reference model. The architecture can be divided into three major parts: (i) VLC Specific consisting of: Physical (PHY) Layer's transmitting source, lighting panel interface, receiver, and error correction, framing of upper layer. (ii) VLC Adaptation consisting of: Media Access Control (MAC) Layer's management and control, services and monitoring, and (iii) ITS Common consisting of the upper layer of TCP, IP and Application which are common to ITS architectures. Each layer is responsible for performing specific tasks and offers services to the higher layers.

The PHY Layer defines the electrical and physical specifications of the devices. The functions and services of this layer are link establishment and termination of a connection to a communication medium. The PHY layer can be divided into two sub-layers.

The lower layer may comprise light source and lighting panel which must support a wide range of vendors. The communication medium being light wave offers wireless line-of-sight (LoS) link in the visible spectrum.

The upper layer is responsible for frame generation, modulation and error handling. Line coding such as 4B5B, 4B6B, NRZ, Manchester [6] can be used depending upon the data to be transmitted.

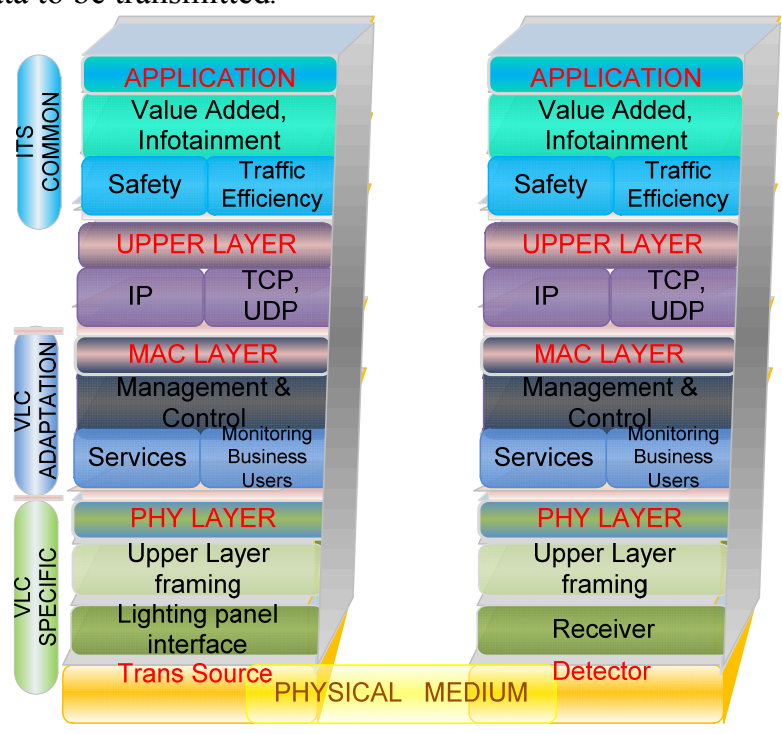

Fig. 2. VLC Reference Model Architecture.

The MAC data communication protocol sub-layer provides addressing and channel access control mechanisms that make it possible for several terminals to be connected. The MAC layer handles all access to the physical visible light channel and supports different applications. It should support visibility, dimming, brightness, signboard for broadcast, security and other related issues. Different data transmission modes such as single mode, (single packet data unit (PDU)) burst mode, packed (multiple data unit) must be supported.

The MAC subsystem interfaces with the upper layer via control and data signaling. The MAC subsystem performs various functions including classification and distribution of control and traffic packets for interfacing with the upper layer. The MAC sublayer should provide beacon management and frame validation in the broadcast mode. VLC technology at some point may be used for accessing value added services. Therefore, a Service sublayer can offer services to the requester. To this end, the layer monitors and interacts with environment as well as RSUs. The business level collects processes and stores the information delivered from several different sources. In addition, the sublayer should provide hooks for implementing application-appropriate security mechanisms. 
EMITTER

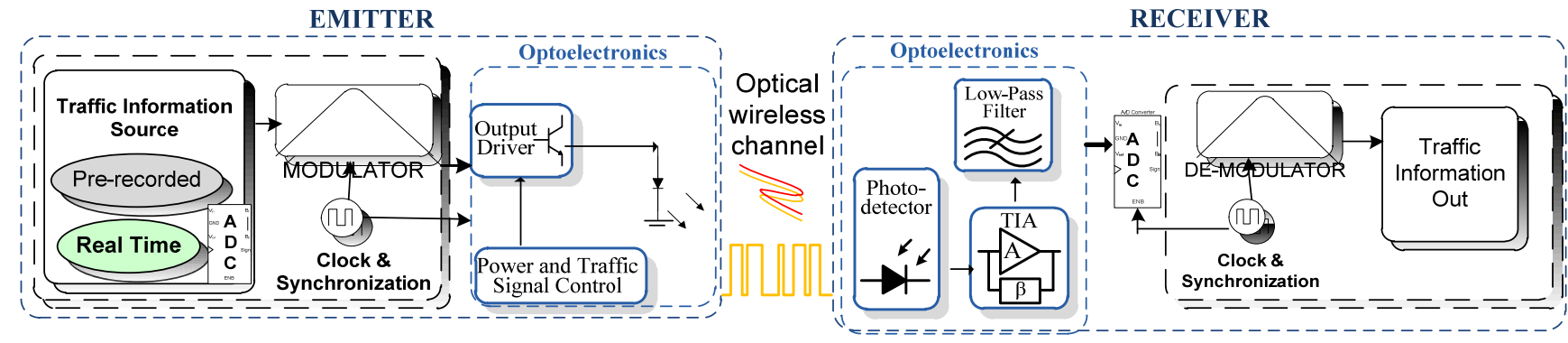

Fig. 3. VLC Information Broadcast System.

Upper layer comprises TCP/UDP/Others, Internet Protocol (IP) and the Application. These are common to ITS architectures. The TCP and IP protocol suite are able to offer a unifying layer between various physical communication technologies and various types of applications used in different contexts and environments. For communication support, application support, service announcement and so on, facility layer is included into the model while safety related, efficient traffic relay and value added dedicated applications are handled using application layer. A security layer monitors and offer authentication for extended services and applications while management and control layer becomes necessary for the reference model for proper control and operation of various components.

Based upon these concepts and highlighting PHY and MAC, a prototype VLC has been developed and tested in different environments. Therefore, we discuss briefly the architecture for VLC information broadcast system in the next section.

\section{VLC ARCHITECTURE FOR INFORMATION BROADCASTING}

VLC is a fast-growing technology able to provide data communication using low-cost and omni-present LEDs and photodiodes. The architecture presented is a VLC broadcast system. The block diagram (Fig. 3) representation along with brief description of transmitter and receiver is presented.

\section{A. The VLC Transmitter}

A VLC Transmitter (emitter) is an electro-optical transducer device that transmits information using visible light waves over wireless transmission medium. VLC systems have become a more viable technology for the future of wireless data transmission, in large part due to the developments in the area of solid-state lighting.

The traffic information either picked in real time or pre-recorded gets modulated with the purpose to switch the LEDs at the expected rate of data transmission. The modulation method used must offer high robustness to background light and at the same time, light should be as bright as possible. Intensity modulation/direct detection (IM/DD) often used in IR can also be used in VLC. Other option is pulse position modulation (PPM), which specifies the modulation of information on the light carrier. This way information is formatted before applying to the carrier. Spread Spectrum is another technique that can be considered. It increases resistance to interference and jamming, and also allows the establishment of secure communications.
In a practical aspect, the modulator also receives information from the traffic control unit so that it can hold information while the light colour changes. This ensures: (i) there is no transmission in the brief period of change in traffic signal; and (ii) transmission is synchronized. The resulting signal is then used to control the switching of the LED through the output driver. The output driver combined with the control signal should ensure sufficient optical power, in order to achieve the expected range of communication. Sometimes the electrical characteristics of the different colour LED's, like the maximum forward current or the forward voltage, might imply the usage of an output driver with distinct channels and a slightly different switching.

The signal processing blocks can be implemented and integrated in field programmable gate array (FPGA) for better data processing capabilities, and it also makes upgrading easier. In the front-end electronics, integrating the LED matrix is essential to specify power consumption, optical range, and maximum operating frequency.

VLC data rate is limited by the switching speed of the emitter LEDs. On the other hand, long distance communication is limited by the transmitted power and background light sources.

\section{B. VLC Receiver}

The VLC receiver is an optic-electronic transducer that receives information, previously modulated in the visible light spectrum, and converts it into electrical signal capable of being processed by a demodulator-decoder. The correct design of this device is crucial to ensure good performance of the overall VLC system. Among other concerning factors are the presence of low-level signals and high noise interference.

The visible light pulses, originated at the system's emitter, are collected in a photo-detector; an optical IR cut-off filter is a viable solution for eliminating unwanted spectral content. Reversely biased photodiode operates in the photoconductive mode, generating a current proportional to the collected light. This current has small values thus requiring pre-amplification to convert it into a voltage. This preamplifier should have low distortion and a large gain-bandwidth product. Transimpedance amplifiers represent the best compromise between bandwidth and noise for this kind of applications. The resulting voltage is then applied to a low-pass filter to remove any high-frequency noise. The signal is then further amplified in the final voltage amplifier stage. Also, DC signal filtering is applied at the input of the amplifying and filtering stages, which helps reduce the DC noise component of the captured signal as well as low-frequency components. The final voltage signal should correspond to the received light pulses which are then decoded in the final decoder block, thus 
extracting the digital data. A practical down-conversion technique that can be considered is direct detection. Clock recovery is necessary to synchronize the receiver with the transmitter. In addition, the system will also need the protocol management unit and data/clock recovery block for the synchronization of received packets.

The detector is characterized by the parameter field of view (FOV), responsivity, and the area. For a larger service area, a receiver with a wider FOV is preferable. However, a wider FOV leads to performance degradation because of possibilities of receiving unwanted light signals. Among the received signals there are also many undesired noise components, which are processed simultaneously.

\section{VLC Channel}

One of the stern requirements of VLC is direct line-of-sight (LoS) especially in outdoor like ITS. The emitted light from LED carries data information in wireless medium. Thus, the intensity of light of the emitter becomes an important parameter on which range of transmission depends. There are many external light noise sources such as Sun light, road/street lights. These are the major issues to be considered in link design. They deteriorate/deceive the intensity of emitter light and may cause false triggering of the photo diode. Optical filters, IR filter should be used to minimize this effect.

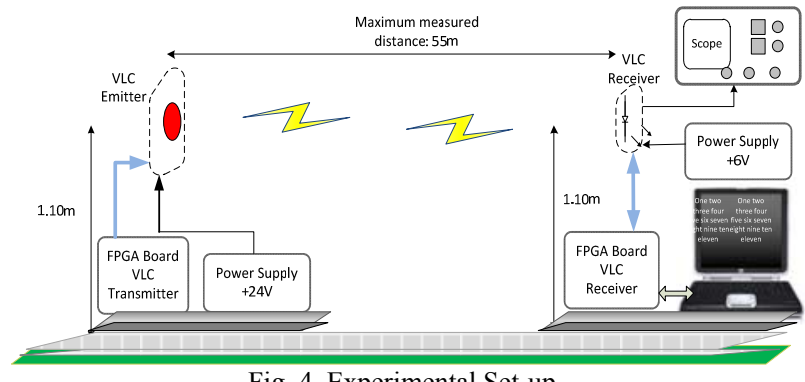

Fig. 4. Experimental Set-up.

\section{EXPERIMENTAL RESULTS}

This section describes experimental description and results from a case study. A number of experiments were carried out in different environments and set-ups. However, only brief description of the scenario is presented in this paper (limitation of length of the paper). Experimental set-up for one of the scenario in outdoor is presented in Fig. 4. Experiment is performed from own designed LED traffic light of standard size $200 \mathrm{~mm}$ in which 240 high brightness (12000mcd intensity) LEDs are used with concentric ring arrangement. The detail can be found in reference [9]. A service area of upto $60 \mathrm{~m}$ was considered and height of emitter and receiver was fixed at $1.50 \mathrm{~m}$ without inclination of any of these devices.

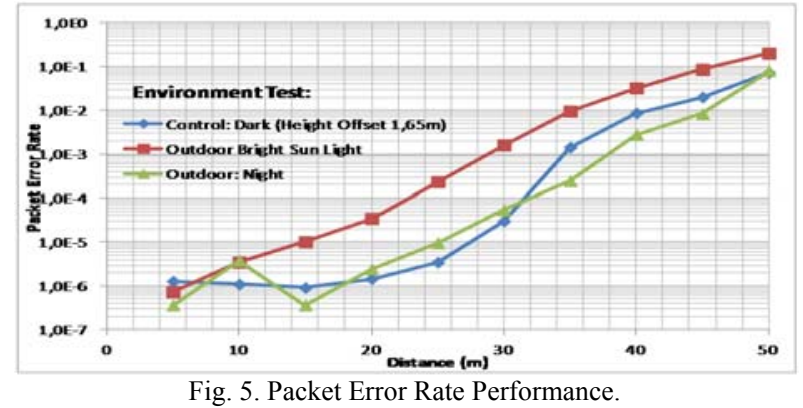

Fig. 5. Packet Error Rate Performance.
Results from three different settings in outdoor environments are presented. These settings are: i) controlled environment (without light interference); ii) night time under $\mathrm{road} / \mathrm{street} \mathrm{lights;} \mathrm{and} \mathrm{iii)} \mathrm{daylight} \mathrm{measurement} \mathrm{under} \mathrm{bright}$ sun light. The transmission rate is $200 \mathrm{kbps}$ while receiver samples at the rate of $1 \mathrm{Mbps}$. The emitter was configured to send 20000 messages containing 50 bytes of random data. On the other hand, the receiver was prepared to store statistics on the number of messages received and the number of packets with and without errors. After collecting a total of 5 million packets, the number of wrong packets received was recorded. Fig. 5 presents packet error rate for different scenarios over distance. It is observed that during day time under bright sun light the performance degradation is almost linear, while during night times, packet error rate variations are found, due to the local nature of the artificial lights. It has also been observed that the performance degrades when measurements are taken directly under the influence of high intensity $\mathrm{road} / \mathrm{street}$ lights. This effect is illustrated in Fig. 6 in terms of percentage of received messages. It can be seen that upto around $35-40 \mathrm{~m}$ of service area (distance), receiver is able to receiver over $60 \%$ of sent messages.

Therefore, for a broadcasting system which has low data rate broadcast feature and where messages are repetitive at regular interval, the above results are found to be satisfactory. Also, considering this data rate, a small message can be repeated many times even on green signal condition of traffic light when a vehicle is in motion at certain speed. This can be described as under.

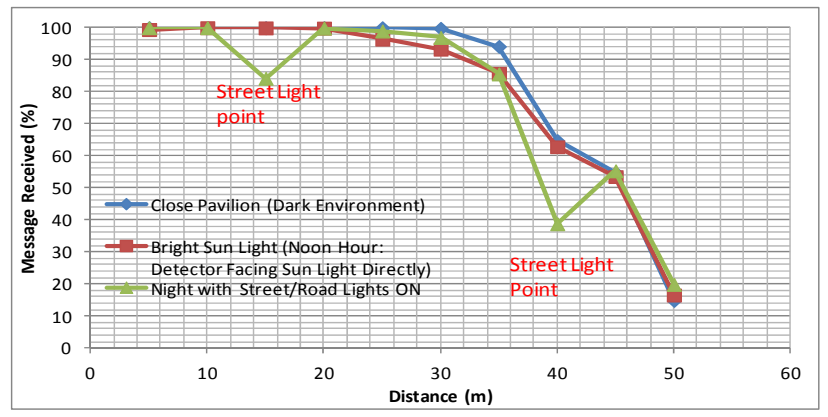

Fig. 6. Effect of high intensity lights on percentage of received messages.

We can calculate the amount of received traffic information over the service area (SA). This is evaluated for different signal-to-noise ratio (SNR) values considering receiver to be in motion at defined speed. It is essential to ascertain that receiver is able to receive necessary information in worst case scenario and that transmitted data rate is sufficient for the intended application. For the condition that vehicle (receiver) is stopped under red signal assuming that the receiver will have maximum amount of time $\left(T_{\text {red }}=60 \mathrm{~s}\right)$ before signal changes. We also assume that the vehicle is running with a speed $\left(V_{c a r}\right)$ of $50 \mathrm{kmph}$ when signal condition remains green. The total service area is considered to be $60 \mathrm{~m}$ while data transmission rate $200 \mathrm{kbps}$ is assumed. Under green signal condition, the receiver remains in motion and therefore, it will have approximately $4.32 \mathrm{~s}$ $\left(T_{g n}=S A / V_{c a r}\right)$ to receive information data. Since, the receiver is moving from far end of SA towards the traffic light (the emitter), it will experience higher SNR values. We assume four different SNR values and calculated successfully received data within received time period $\left(T_{g n}\right)$ for four 
discussed modulation schemes [9].

The received information data is given as:

$$
N_{r x}=T \times R_{b} \times(1-\mathrm{BER})
$$

where, time $(T)$ can be either $T_{g n}$ or $T_{\text {red }}$ for the remaining time for the receiver to receive data during traffic signal condition of green and red respectively, $R_{b}$ is the data transmission rate and BER is the bit-error-rate of the modulation scheme.

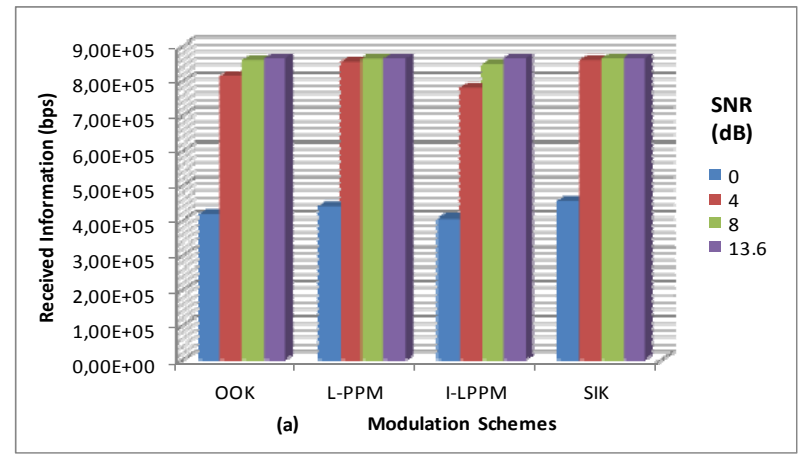

Fig. 7. Received Information for different modulations when receiver is in Motion.

Received information bits performance over service area for different modulation schemes is depicted in Fig. 7. In this case, the receiver is moving from far end of the SA to the traffic point. As receiver moves from longer distance to close to the traffic point, the information received will be higher because of increasing SNR values. The amount of received data for the four different modulation schemes for the different SNR values is shown. As expected, DSSS SIK performs better than any other modulation schemes described here. SNR value of $13.6 \mathrm{~dB}$ is selected so that a BER of $1 \mathrm{e}-6$ is obtained for the case of basic OOK operation. Though, a small difference in the received information in some cases is found, however, BER performance is very high. In the case of stationary receiver, the amount of received information will be higher.

\section{CONCLUSION}

Emerging VLC systems have many prospective novel and challenging applications especially in outdoor. A traffic information broadcast system for road safety applications in ITS is one example which has been discussed in this paper. A conceptual integration method of VLC into ITS along with reference model is presented. The results from experiments of case study show that VLC is suitable for intended application.

\section{ACKNOWLEDGMENT}

Author would like to thank colleagues Nuno Rafael and Domingo Terra for their efforts in experimental verification.

\section{REFERENCES}

[1] O. L. Davies. (September 2012). World Health Organization Media Centre. [Online]. Available: http://www.who.int/mediacentre/factsheets/fs358/en/index.html

[2] Wireless Access in Vehicular Environment (WAVE), IEEE, 2010.

[3] G. Davis and B. McKeever (July 2006). Research for V2I Communication and Safety Applications. ITE Technical Conference [Online] Available: http://www.its.dot.gov/presentations/pdf/V2I_Safety2011 ITE_Techn ical_Final.pdf.

[4] M. Schulze, D. AG and T. Makinen. (January 2008). Preventive and Active Safety Applications Integrated Project [Online]. Available: http://prevent-ip.org/wp-content/uploads/62008.pdf

[5] Intelligent Transportation Systems- Communication Access for Land Mobile (CALM), ISO 21213, 2008

[6] M. Akanegawa, Y. Tanaka, and M. Nakagawa, "Basic study on traffic information system using LED traffic lights," IEEE Trans. On Intelligent Transportation System, vol. 2, pp. 197-203, 2001

[7] B. W. Haswani, K. Toshihiko, H. Shinichiro, and N. Masao, "Visible Light Communication with LED Traffic Lights Using 2-Dimensional Image Sensor," IEICE Trans. Fundamentals, vol. E89-A, 2006.

[8] R. Bossom, R. B. T. Ernst, and T. Cosch, (March 2009). European ITS Communication Architecture Overall Framework Proof of Concept. [Online]. Available: http://www.comesafety.org/uploads/media/COMeSafety_DEL_D31_ EuropeanITSCommunicationArchitecture_v2.0_01.pdf

[9] N. Kumar, "Visible Light Communication for Road Safety Applications," $\mathrm{PhD}$ Dissertation, Dept. of Electronics Telecommunication and Informatics, University of Aveiro, Portugal, Dec. 2011

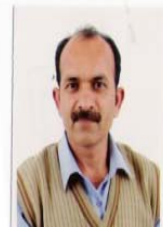

Navin Kumar became member of IEEE in 2006. He obtained his undergraduate degree in electronics and telecommunication engineering from Delhi, India in 1996, Master of Engineering in digital system engineering from Motilal Nehru national Institute of technology, Alllahabad, India in 2000 and Doctoral degree in telecommunication from University of Aveiro, Minho and Porto, Portugal in 2011.

He has worked in Indian Air Force as Graduate Engineer until 2003 and from 2003 to 2007 as Expatriate Faculty with Addis Ababa University, Ethiopia. He secured foundation of science and technology (FCT), govt. of Portugal research grant towards his $\mathrm{PhD}$ (2008-2011). He continued his research work after $\mathrm{PhD}$ at the Institute of Telecommunication, Aveiro, Portugal. Currently, he is working as Professor at the dept. of Electronics and Communication engineering, the Oxford College of Engineering, Bangalore, India. He has authored a book and few journals and conference proceedings. His research interest is optical wireless communications, intelligent transportation systems, vehicular communications, cloud computing and next generation networks. Dr. Kumar is a member of IET (UK), IAENG (HK), Fellow of IETE and IE (India). He is also C Eng. (IE India). He has won Fraunhofer challenge award for his thesis work in 2011 and Gowri Memorial award for the best paper in 2008-2009. 